Farm animal welfare - who writes the rules?

Occasional Publication No. 23 - British Society of Animal Science 1999

edited by A. J. F. Russel, C. A. Morgan, C. J. Savory, M. C. Appleby and T. L. J. Lawrence

\title{
The stunning monitor for cattle, sheep and pigs
}

\author{
J. Heal \\ Meat and Livestock Commission. Winterhill House, Snowdon Drive, Milton Keynes, MK6 1 AX
}

\section{Introduction}

A survey conducted by the Division of Food Animal Science (DFAS) of Bristol University on behalf of the Ministry of Agriculture Fisheries and Food (MAFF) looked at the electrical stunning of pigs in British abattoirs. The survey concluded that most abattoirs were using equipment with low voltage capabilities and that many were unable to meet the minimum current requirements required by EU legislation. MAFF was therefore reluctant to implement legislation within Great Britain (GB) before equipment was available to allow the industry to meet the minimum requirements.

Following the results of the survey, the Meat and Livestock Commission (MLC) sponsored a research project to develop a system of controlling, monitoring and recording the delivery of stuns to pigs. The system would be designed to calculate, by checking the voltage available and measuring the impedance of the animals head, if an effective stun could be delivered. The system would only allow the operator to proceed with the stun if predetermined parameters were met and would therefore provide a fail-safe function as its primary rôle.

\section{Stunning monitor development}

Extensive field testing with prototypes resulted in the development of a unit that incorporates an effective fail-safe function. Additionally, a visual display of each stun profile provides an on-line monitoring capability for abattoir personnel, Meat
Hygiene Service (MHS) and MAFF State Veterinary Service (SVS) staff. A further feature is a record of the profile of every stun to allow plant management and officials to view stuns retrospectively ensuring requirements are met throughout all operational periods.

The stunning monitor is now commercially available, manufactured by Hellenic Systems Ltd (HSL) and marketed by the MLC. The unit is compatible with all existing stunning equipment and therefore allows an abattoir to provide quickly this important element to monitor operations on the slaughter line. MAFF is considering the draft GB legislation for all three species as the stunning monitor is designed to be used for cattle and sheep as well as pigs. A legislative implementation date will be set to allow abattoirs a reasonable lead time to install the monitoring equipment.

The installation of the stunning monitor will ensure that only effective stuns are delivered to each animal through the fail-safe mechanism. This control will act as a training tool for stunner operatives, raising performance standards. The recording of all stun profiles will provide visibility of operations to MHS and MAFF personnel who have responsibility to ensure welfare standards are met. The equipment will make a significant contribution in providing reassurance to retailers and consumers that welfare standards are maintained at the highest level in a most critical area of the processing chain. 